\title{
LA SOLIDARIDAD INTERNACIONAL CON LA REPÚBLICA EN GUERRA
}

\section{LAURA BRANCIFORTE (UC3M)}

Universidad Carlos III de Madrid

$\underline{\text { lbranciforte@hum.uc3m.es }}$

\begin{abstract}
Resumen:
En este artículo se aborda el tema de la solidaridad internacional a partir de la organización kominterniana del Socorro Rojo Internacional (SRI) que nació en Rusia en 1922. Se traza la acción de este organismo en España tras la revolución de octubre de 1934, cuando asumió una gran importancia social y política y se convirtió en un gran "movimiento solidario". A partir de este momento y durante 1935 la ayuda humanitaria, las campañas de solidaridad o la recogida de fondos en el extranjero hicieron del SRI una pieza clave frente a la represión, las mujeres lograron un protagonismo absoluto, y empezaron a desarrollar funciones de tipo social y político. El SRI se iría "feminizando" en sus bases, aunque menos en su dirección, como era de esperarse. Durante la guerra civil se convirtió en una de las más importantes organizaciones del antifascismo femenino y en una pieza clave del sistema sanitario militar republicano.
\end{abstract}

Palabras clave:

Ayuda - Solidaridad - Represión - Mujeres - Acción Sociopolítica

\begin{abstract}
:
In this article, we explore international aid starting from the Kominternian organization focusing our attention on the International Red Aid (SRI) which was born in Russia in 1922. We are particularly concerned with the actions developed by this organism in Spain after the October revolution in 1934, when it assumed the characteristics of a huge "solidarity's movement". Since October 1934 the humanitarian aid, the campaigns of solidarity, the recollect of economics' resources from abroad had transformed the SRI into a key piece against the repression. Women became protagonists of the SR' labor and started developing social and political functions. The SRI began feminizing its bases, though its leadership was not affected by the change, as it was expected. During the civil war the SRI finally became the most important organization of feminine antifascism and the base of the Republican military health system.
\end{abstract}

\section{Keywords:}

Aid - Solidarity - Repression - Women - Social and Political Action 


\section{LA SOLIDARIDAD INTERNACIONAL CON LA REPÚBLICA EN GUERRA}

\section{LAURA BRANCIFORTE (UC3M)}

lbranciforte@hum.uc3m.es

\section{Introducción}

El objetivo de este artículo es dar a conocer una organización que formó parte del más amplio movimiento internacionalista comunista y que, pese a sus declaradas finalidades y alcances humanitarios y solidarios, estuvo condicionada por haber nacido bajo el paraguas de la Komintern, hecho que dificultó el reconocimiento de su acción solidario-humanitaria. Por supuesto, con el Socorro Rojo Internacional (SRI) no nos encontramos, tampoco, frente a una de las "agencias humanitarias imparciales e independientes" que intervinieron en la ayuda humanitaria en la guerra civil (Cruz Roja, Save The Children Fund, Save The Children International Union, o el Servicio Civil Internacional y la Comisión Internacional) que han sido recientemente abordadas con profundidad en un libro escrito por Gabriel Petrus ${ }^{1}$. Por el contrario, nos topamos de lleno con una organización que nace de la necesidad de la internacionalización y, al mismo tiempo, de la difusión de la solidaridad y de la propaganda comunista. Estos dos últimos términos - propaganda y solidaridad- se pueden encontrar fácilmente enfrentados y, quizás, hayan dificultado que se le haya dedicado más espacio como una organización autónomacon un destacado papel en la ayuda humanitaria.

El discurso de politización de la solidaridad es válido y está presente sobre todo en la década de los veinte, cuando la faceta del SRI es más política y su finalidad como international networking de la Tercera Internacional ${ }^{2}$ se concreta en la constitución de redes clandestinas o, mejor todavía, en la formación de grupúsculos de oposición y de resistencia al fascismo en Europa. La represión del SRI empezó en Italia, donde la persecución del Soccorso Rosso fue despiadada y se vivieron las peores condiciones de clandestinidad de las secciones nacionales de la organización hasta la aplicación de las leyes de 1926 que determinaron su inmediata disolución ${ }^{3}$, incluso antes de la ilegalización del PCI (Partido Comunista Italiano) ${ }^{4}$.

En la limitada consideración historiográfica del SRI también hay que tener en cuenta que, como lo destaca Paul Preston en la introducción al libro de Gabriel Petrus, "la ineludible atención de la mayoría de los historiadores sobre los aspectos económicos, políticos o militares de la guerra española ha relegado al olvido temas importantes como el estado de la población más allá de las líneas de combate" ${ }^{35}$. A este último aspecto, por supuesto, se dedicó el SRI en situaciones de conflicto.

\footnotetext{
1 Petrus, Gabriel, La ayuda humanitaria (1936-1939), Granada, Comares, 2015.

2 García, Hugo; Yusta, Mercedes; Tabet, Xavier y Clímaco, Cristina (eds.), Rethinking Antifascism, Berghahn Books, New York, 2016, pág. 84.

${ }^{3}$ Soccorso Rosso Internazionale, Sezione Italiana, Direttive per $i$ Comitati di base Patronati fiduciari e Attivisti, en Archivio Fondazione Istituto Gramsci (AFIG), Fondo Soccorso Rosso (1936), fasc. 1423. Desde 1923 hasta 1925 la organización italiana vivió en la semilegalidad, y desde 1925 pasó a la más completa ilegalidad. «Riunione del Comitato Centrale del Soccorso Rosso Sezione Italiana», en AFIG, Fondo Mosca, fasc. 215.

4 Agosti, Aldo, Storia del PCI, Roma Bari, Laterza, 2000, págs. 26-27.

${ }_{5}^{5}$ Petrus, Gabriel, La ayuda humanitaria..., op.cit. pág. X.
} 
En el menor interés en el ámbito de la historiografía suscitó el movimiento comunista internacional pudo influir también -como lo subraya Claudio Natoli- la tendencia profundamente arraigada en los historiadores occidentales de considerar a las organizaciones auxiliares de la Komintern como meros apéndices de la política de la Internacional Comunista (IC). De esta última consideración no se exime tampoco, según este mismo autor, la historiografía soviética y aquella de los países del este ${ }^{6}$.

Ahora bien, es preciso reconocer que la bibliografía producida en los últimos años sobre el antifascismo -o más bien los antifascismos ${ }^{7}$, dado que entre 1934 y 1935 en el seno de la izquierda se difundieron diferentes formas de entender este "sentimiento" $-{ }^{8}$, ha ido aumentando en cantidad y calidad. Pienso en la amplia bibliografía sobre el antifascismo en Italia, en Francia, en Alemania o en compendios más generales que, sin embargo, todavía parecen poner en duda la presencia de estudios acreditados sobre el tema?. Destaca también el florecimiento de una amplísima bibliografía sobre el asociacionismo antifascista femenino. Todas estas aportaciones, desde distintos puntos de vista, han venido gradualmente mencionando e incluyendo al SRI.

En España la atención al Socorro Rojo $(\mathrm{SR})^{10}$ se ha circunscripto a referencias a él en estudios locales o regionales, eso sí de gran utilidad ${ }^{11}$, algunos focalizados en la historia asturiana ${ }^{12}$, que adquieren cierta importancia informativa por lo que respecta al papel principal que tuvo el SR en Asturias a partir de 1934. Finalmente, en relación con los estudios dedicados al SRI cabe mencionar la importancia de aquellos que han reservado un espacio importante a la organización a través de las biografías de algunos o, más bien, de algunas de las mujeres que fueron sus principales protagonistas. Las reconstrucciones profundas de perfiles biográficos de las mujeres antifascistas que tuvieron un papel fundamental en su internacionalización, han permitido ahondar en los roles femeninos en el Socorro Rojo en España ${ }^{13}$. Otra novedad historiográfica es la reciente contribución a la reconstrucción de algunos de los perfiles de las brigadistas

\footnotetext{
${ }^{6}$ Claudio, Natoli, "Tra solidarietà e rivoluzione: il Soccorso Rosso Internazionale”, en Riccardo Toffoletti (coord.), Tina Modotti una vita nella storia,Udine, Arti Grafiche friulane, 1995, pág. 193-211, pág. 193.

7 Serge Wolikow, "Les gauches, l'antifascisme et le pacifisme pendant les années 1930", en Jean-Jacques Becker et Gilles Candar (coord.), Histoire des Gauches en France, XXe Siècle: à l'épreuve de l'histoire, vol. 2, París, Ed. La Découverte, 2004.

${ }^{8}$ Laura Branciforte, "La solidaridad bajo el lema del antifascismo", en $\mathrm{M}^{\mathrm{a}}$ Candalaria Fuentes Navarro et alii (eds.), II Encuentro de Jóvenes Investigadores en Historia Contemporánea, Granada, Universidad de Granada, 2010.

${ }^{9}$ En la contraportada del libro de Michael, Seidman, Antifascismos, 1936-1945.La lucha contra el fascismo a ambos lados del Atlántico, Alianza, Madrid, 2017, se lee, refiriéndose al antifascismo, "ningún historiador ni científico social ha intentado definir su propia historia y naturaleza".

${ }^{10}$ Para mayor brevedad y claridad nos referiremos a la sección española del SRI en España con la sigla de SR.

${ }^{11}$ Carmen González Martínez, Guerra Civil en Murcia. Un análisis sobre el poder u los comportamientos colectivos, Murcia, Universidad de Murcia, 1999; Lucía Prieto Borrego y Encarnación Barranquero Texeira, Población y Guerra Civil en Málaga: Caída, éxodo y refugio, Málaga, Servicio de Publicaciones de la Diputación Provincial de Málaga, 2007, págs. 224-229; Sofía Rodríguez López, Mujeres en guerra. Almería, 1936-1939, Sevilla, Mojácar (Almería), Fundación Blas Infante y Arraéz Editores, 2003.

12 Taibo II, Paco Ignacio, Historia General de Asturias, t. 8; Erice, Francisco (coord.), Los comunistas en Asturias 1920 1982, Gijón, Trea, 1996.

${ }^{13}$ Sobre Matilde Landa: Ginard I Feron, David, Matilde Landa. De la Institución libre de enseñanza a las prisiones franquistas, Flor de Viento Ediciones, Barcelona, 2005; Morán, Gregorio, Miseria y grandeza del Partido Comunista de España 19391985, Planeta, Barcelona, 1986, pág. 37; Di Febo, Guiliana, Resistencia y movimiento de mujeres en España: 1936-1976, Barcelona, Icaria, 1979, págs. 58-62; Sobre Tina Modotti: Branciforte, Laura, "Tina Modotti: un' appassionante biografía nell' antifascismo europeo. Spagna, 1933-1939" en Paolo Ferrari, Claudio Natoli (coord.) Tina Modotti, Arte e Libertà fra Europa e Americhe, Forum, Udine, 2017, págs. 117-130; Sobre Encarnación Fuyola: Nash, Mary, Rojas, Las mujeres republicanas en la guerra civil, Taurus, Madrid, 1999, págs. 76-88; Branciforte, Laura "Encarnación Fuyola: del internacionalismo antifascista al exilio a México" en Cenarro, Ángela e Illion, Régine (eds.): Feminismos. Contribuciones desde la historia, Colección Estudios Feministas, Prensas de la Universidad de Zaragoza, Zaragoza, 2015.
} 
internacionales que participaron en la guerra civil, algunas de las cuales también trabajaron y se afiliaron al SRI ${ }^{14}$.

Tras esta breve reflexión sobre la presencia del SRI en la historiografía, me gustaría centrarme en su labor, en su contribución a la defensa de la República y en su fuerte relación con el antifascismo internacional en el cual se insertó y del cual el SR se convirtió en uno de sus principales promotores.

\section{El SR: de la dictadura de Primo de Rivera a los inicios de la II República}

En este artículo no nos ocuparemos de la labor que el Socorro Rojo desarrolló en España en sus comienzos cuando, desde 1923, ejerció como organismo clandestino, todavía de diminutas dimensiones, con fines sobre todo de propaganda y oposición política a la dictadura militar de Primo de Rivera. Simplemente delinearé las líneas de actuación que antecedieron y fueron preliminares a la política del SR en los años de la II República y que le valieron para ir sentando las bases de su difusión posterior en la década de 1930 en el territorio nacional.

La organización kominterniana sufrió el mismo destino y desarrollo que el Partido Comunista de España (PCE) que se caracterizó desde el principio por las divisiones y el aislamiento casi total con respecto a las demás fuerzas políticas ${ }^{15}$. El SR tuvo que organizar e implantar su Comité Central y sus federaciones regionales en la clandestinidad padeciendo continuas detenciones de sus dirigentes y escasos afiliados. La virulencia de esta represión impidió la extensión del PCE y del SR y supeditó su supervivencia a la más completa clandestinidad.

El SR desde 1923 hasta 1931 estuvo sujeto, principalmente, a las dinámicas políticas de un partido minoritario (por esas fechas contaba con 120 militantes en todo el país) y a los objetivos fijados por la Komintern. Pese a ello, la organización logró difundirse por todo el territorio nacional español por medio de los comités regionales y locales, aunque de forma heterogénea e irregular. Consiguió, asimismo, crear un estrecho canal preferente de comunicación con el Secour Rouge Français (SRF) y con el Bureau Latin. Esta conexión fue precursora de una colaboración, no siempre fácil y que, no obstante, dio enormes frutos a partir de 1934.

La capacidad del Socorro Rojo para superar las imposiciones de políticas estrictamente partidistas marcadas por la política de frente único, después del VI Congreso de la Komintern en 1928, le permitió encontrar su espacio de difusión entre la población española y poner en práctica el "dictamen" internacionalista del SRI que se basaba en el ideal imperativo de "sin diferencias de partidos ni tendencias" y en una supuesta y aparente "aconfesionalidad". La función asistencial con la cual nació el SRI justificó desde sus inicios su aparente carácter y operatividad apartidista y le permitió alcanzar un consenso más amplio que el PCE tanto en la dictadura como durante la República. Llegó a tener Comités regionales y provinciales (en concreto, ocho federaciones regionales: la valenciana, la andaluza, la galaica, la catalana, la vasco-navarra, la castellana, la asturiana y la del Duero, en vía de organización y cuyos comités regionales residían respectivamente en sus ciudades principales ${ }^{16}$ ) de forma difusa en el territorio nacional pero que denotaban la propagación de un sentimiento cada vez más extendido de la necesidad de su constitución. Por lo general, se trataba de comités muy reducidos, pero a veces numéricamente

\footnotetext{
${ }^{14}$ Cantaluppi, Augusto y Puppini, Marco, Non avendo mai preso un fucile tra le mani: antifasciste italiane alla guerra civile spagnola: 1936-1939, AICVAS, Milano, 2014.

15 Véase Meaker, Gerald H., La izquierda revolucionaria en España 1914-1923, Barcelona, Ariel, 1978, pág. 551 y ss.

16 "Barcelona, Valencia, Sevilla, Madrid, Oviedo, Bilbao, Santiago y Palencia". "Plan general de reorganización de la sección española del Socorro Rojo Internacional; de sus Comités responsables y del servicio de control”, 27 de mayo de 1926, en Archivo Histórico del PCE (AHPCE), film I, apartado 23, pág. 2.
} 
más importantes que los del propio Partido Comunista y que en términos totales hacían inclinar netamente la balanza de los afiliados hacia el $\mathrm{SR}^{17}$.

Con la llegada de la II República no podemos decir que el Partido Comunista o el SR vivieron condiciones idílicas para su difusión, con una República "atemorizada" por los extremos y centrada en su defensa y en su apuntalamiento sobre una base reformista y moderada. A pesar de las limitaciones impuestas al Partido Comunista, al que la República llegó a perseguir a partir de 1933 de forma sistemática ${ }^{18}$, el SR pudo seguir desarrollando su propaganda y sus actividades sin grandes impedimentos y tal vez incluso con mayor libertad de acción, porque no estuvo directamente perseguido hasta 1934. A partir de este año el SR, acostumbrado como el mismo PCE a la ilegalidad, tomó todas las "medidas técnicas" para desarrollar su trabajo en estas condiciones $^{19}$, así como para intentar legalizar su labor.

Desde los primeros años de la República, el SR comenzó a expandir su red solidaria y a experimentar nuevas modalidades de acción acordes con la mayor apertura política, poniendo fin a las divisiones y conflictos internos que habían caracterizado sus orígenes. Se mostró como un eficaz canal político complementario al Partido y a la política kominterniana, que en estos años, sobre todo desde 1932, acorralaba a sus secciones nacionales por medio de la acción de sus delegados ${ }^{20}$.

Durante el primer bienio republicano, la organización fue aumentando en miembros a la vez que el PCE. Como ya había sucedido en los años primorriveristas, la organización de solidaridad kominterniana gozó de cierta superioridad numérica respecto del Partido, registrando en 1932, el año del Congreso del PCE, el triple de afiliados. En dicho año, el SR contaba con un total de 33.100 afiliados, 10 comités regionales y 213 comités locales $^{21}$. Las cifras del Partido oscilaban entre los 10.500 y 12.000 afiliados, lo que supuso un aumento exponencial nada más empezar la República ${ }^{22}$.

En este año de 1932 se celebró en Madrid, el 12 de octubre, el primer Congreso Nacional del $\mathrm{SRI}^{23}$, una fecha tardía respecto a su fundación y a las demás organizaciones europeas (como el

\footnotetext{
${ }^{17}$ Los afiliados en 1926 llegaban a 2.000 "en números redondos" frente a las pobres cifras de afiliación al PCE que, en 1928, apenas contaba con 777 afiliados. AHPCE, film I, apartado 23, p. 2 y Bizcarrondo, Marta y Elorza, Antonio, Queridos Camaradas. La Internacional Comunista y España 1919-1939, Barcelona, Planeta, 1999, pág. 64.

18 Cruz, Rafael, "Del partido recién llegado al partido de todos. El PCE 1920-1939”, en Bueno, Manuel; Hinojosa, José y García, Carmen, Historia del PCE, I Congreso 1920-1977, op. cit., pág. 143-158, p. 149. Cabrera, Mercedes, La patronal ante la II República: organización y estrategia, 1931-1936, Madrid, Siglo XXI, 1983; Aróstegui, Julio (ed.), La República de los trabajadores: la Segunda República y el mundo del trabajo, Madrid, Fundación Francisco Largo Caballero, 2006, Cruz, Rafael, El Partido Comunista de España en la II República, op. cit., pág. 53.

19 "Reunión celebrada con el CEI del SRI el 5 abril 1934", en AHPCE, film X, apartado 132, pág. 2.

${ }^{20}$ Esta situación resultó especialmente evidente en la omnipresencia del italo-argentino Vittorio Codovilla en España (delegado con plenos poderes de 1932 a 1937) Bizcarrondo, Marta y Elorza, Antonio, Queridos Camaradas. La Internacional Comunista y España 1919- 1939, op. cit., pág. 113; Togliatti, Palmiro, Escritos sobre la guerra de España. El testimonio histórico del dirigente extranjero que más influyó en la lucha contra el fascismo en España, Barcelona, Crítica, 1980, pág. 142.

${ }^{21}$ SRI, Normas para el funcionamiento del Socorro Rojo de España, op. cit., pág. 6; los mismos datos se encuentran en SRI, Resoluciones del Primer Congreso Nacional de la SE del Socorro Rojo Internacional, Madrid, 1932, pág. 6; Informe general de la situación del SRI de España, diciembre 1933, en AHPCE, film X, apartado 132.

${ }^{22}$ David Ruiz constata en 1932 la presencia en el PCE de 10.500 afiliados. Según Rafael Cruz, los afiliados oscilaban en abril de 1931 entre 800 y 120 militantes (según las fuentes). En mayo de 1931 eran 3.000; en julio, 6.000; en agosto, 7.000; a finales de año, 8.800. En 1932 se dobló la cifra de finales de 1931. En noviembre, el periódico comunista Mundo Obrero señalaba 17.000 afiliados. Eso induce a pensar que a finales de año el PCE pudo tener entre 12.000 y 15.000 afiliados, como hemos podido constatar en otras fuentes. Ruiz, David, Insurrección defensiva y revolución obrera. El octubre español de 1934, Barcelona, Labor Universitaria, 1988, pág. 73; Cruz, Rafael, El Partido Comunista de España en la II República, op. cit., pág. 58.

23 SRI, Normas para el funcionamiento del Socorro Rojo de España, op. cit., pág. 6; los mismos datos se encuentran en SRI, Resoluciones del primer Congreso Nacional de la SE del Socorro Rojo Internacional, op. cit., pág. 3.
} 
Soccorso Rosso y el Secours Rouge, que tuvieron sus primeros congresos, respectivamente, en mayo de 1925 y en octubre de $1926^{24}$ ). A pesar de su implantación tras un primer Congreso Nacional y el alto número de afiliados, nos encontramos frente a una organización todavía "estancada", a la que se atribuye, con razón, la limitación de que seguía conservando el carácter de un organismo de "socorro mutuo" en el ámbito comunista y de estar vinculado a un sectarismo intrínseco ${ }^{25}$.

Mientras tanto, la evolución de la política de la II República, con el consiguiente cese de la colaboración socialista en el gobierno en septiembre de $1933^{26}$, cambió el rumbo de la vida republicana. Se fue perfilando un nuevo panorama político más radical a la izquierda, aunque todavía las sendas comunista y socialista quedaban muy lejanas. El mismo defensor del "gradualismo reformista", Francisco Largo Caballero, fue conquistado por la vía de la radicalización, pues la consideró la única posibilidad de acción ${ }^{27}$.

Si este fue el marco de referencia política de la acción del SR desde el inicio de la República española, otra fue la política y la fisonomía que dicha organización fue asumiendo en línea con las directivas de la "casa" desde el año 1933, cuando encontró en la política de unidad de acción su función específica. La actividad del SR se fue incrementando en el territorio español tras las elecciones generales de noviembre de 1933 y la constitución del nuevo gobierno del radical Alejandro Lerroux. La organización se cimentó de inmediato en una campaña denigratoria contra la nueva realidad política de la Confederación Española de Derechas Autónomas (CEDA) -que nació en marzo de 1933 y que se caracterizaba por la condición católica de sus miembros y de sus programas-, refiriéndose a ella con lemas y fórmulas recurrentes como el "terror radical-cedista" o el fruto de la "reacción vaticano-fascista" 28 , siguiendo a menudo el empobrecedor uso de la propaganda kominterniana y cierto reciclaje de la terminología que vaciaba de sentido estas declaraciones.

Sin embargo, en concomitancia con el nuevo clima político se constata, ante todo, un aumento progresivo y significativo de la afiliación al Socorro Rojo que, en julio de 1933, alcanzaba su pico máximo de 37.500 afiliados. El dato más significativo en 1933 es que se aprecia una presencia mayor de afiliados sin partido y de distinto origen político, así como una disminución del número de comunistas. En el crecimiento general del SR hay que incluir una duplicación de los comités regionales y provinciales respecto al año anterior, que llegaron en 1933 a un total de 20 comités (16 regionales, 4 provinciales y 368 locales $)^{29}$. El elevado número de comités nos indica una distribución cada vez más extensa en el territorio nacional, si bien no del todo homogénea, con

${ }^{24}$ Véase "iAu sujet du secours rouge international, 22 mars 1926", en Archives Nationales Françaises (AN), série F7, "Secours Rouge" Bureau Latin-13154. Secours Rouge International, 1921-1929; APCF, 3 MI 7/ 56. Secours Rouge Internacional, Fond 539.9, Dossiers 1173: "1 ${ }^{\mathrm{er}}$ congrès de la section française, rapports»; Bollettino de $i$ patronati de lle vittime del Fascismo, relación de febrero de 1928, firma de la Comisión Central, en APCF, 3 MI 7/ 61-1928. Secours Rouge Internacional, Fond 539.3. Dossier 1233.

${ }^{25}$ SRI, Las masas contra el terror 10 años del Socorro Rojo Internacional, op. cit., pág. 19.

${ }^{26}$ La Comisión Ejecutiva del Partido Socialista presentó la dimisión el 7 de septiembre de 1933. Pese al intento de Prieto de esperar, se votó por unanimidad la propuesta de Caballero de declarar "rotos todos los compromisos". Cabrera, Mercedes, "Proclamación de la República, Constitución y Reformas", en Juliá, Santos, (coord.), República y Guerra en España (1931-1939), Espasa Libros, Madrid, 2006, pág. 72.

27 Aróstegui, Julio "Largo Caballero y la construcción de un código de trabajo", en Aróstegui, Julio (ed.), La República de los trabajadores: la Segunda República y el mundo del trabajo, op. cit., pág. 223.

${ }^{28}$ Se habla a menudo de víctimas de la "reacción vaticano-fascista", ¡Combate! Órgano del Socorro Rojo Internacional (SE), abril de 1935, año II, núm. 4 (segunda época), editado por el Socorro Rojo Internacional, pág. 2. Se menciona: "La represión brutal de la reacción 'vaticano-fascista' que deseaba liquidar el movimiento revolucionario". "Tareas inmediatas del SRI", Madrid, 20 de marzo de 1935, firmado por el Comité Ejecutivo Nacional-Secretariado. Se utiliza también el término de "justicia vaticano-fascista”. ;Combate! Órgano del Socorro Rojo Internacional (SE), abril de 1935, año II, núm. 5 (segunda época), 1 de mayo, en AHPCE, film XIV, apartado 171. Octavilla del Socorro Rojo Internacional, "A las masas populares de España”, en AHPCE, film X, apartado 132.

${ }^{29}$ Socorro Rojo Internacional (SE), "Comité Ejecutivo Servicio de Estadística. Relaciones de la represión para el secretariado del Partido Comunista de España”, pág. 1. 
un crecimiento de la presencia de la organización principalmente en las regiones de «Asturias, Vasconia, Guipúzcoa» ${ }^{30}$. Sin duda, el buen funcionamiento del SR en Asturias fue un precedente para la capacidad de movilización que alcanzó la organización en esta región después de los acontecimientos de octubre de 1934.

El SR iba, por tanto, fortaleciendo su actividad, sobre todo cumpliendo una acción de denuncia, protesta y socorro. Hacia 1934, antes de la insurrección de octubre, se percibe ya la puesta en marcha de dos líneas principales de acción: una primera, más específicamente asistencial, destinada al cuidado de la población, y una segunda más declaradamente política. La forma principal con la que la organización pretendió llevar adelante sus objetivos políticos fue mediante la creación de "Comités de frente único", que tendrían que reagrupar a "los presos revolucionarios de todas las tendencias" $"$.

Lo que se puso de manifiesto entre los años 1933 y 1936 fue que -frente a las fuertes reticencias y desencuentros de las ejecutivas socialista, comunista y anarquista respecto a un camino en común- el Socorro Rojo se convirtió en una ágil herramienta para superar los obstáculos a la unidad política, que ni siquiera las políticas aliancistas aplicadas antes y después de octubre de 1934 lograron superar.

\section{La labor político-asistencial del Socorro Rojo tras la revolución de octubre en Asturias}

La metamorfosis en la principal organización volcada en la ayuda y socorro de los detenidos políticos y de sus familias se verificó tras la revolución de octubre de 1934. El compromiso del SR con la población fue algo que la organización hasta ahora nunca había experimentado. Por otro lado, el movimiento de octubre consiguió, de forma indirecta, además del objetivo asistencial, el político: la Komintern y el SRI obtuvieron del SR lo que se esperaban. El SR se transformó en una amplia organización de frente único, en un instrumento real de movilización y, por último, en un medio de intervención de la política comunista nacional e internacional en el territorio español.

En el semestre posterior a octubre de 1934, la acuciante necesidad de organizar las ayudas a presos y represaliados actuó como elemento propulsor para la aglutinación popular que fue, por el contrario, repentina y masiva. La puesta en marcha del socorro a la población asturiana fue inmediata y se dirigió ante todo a la protección de los perseguidos y a la lucha contra la pena de muerte $^{32}$.

En la situación de desorganización que sobrevino después de 1934, el SR, gracias a la considerable presencia de afiliados, se presentó a los demás organismos solidarios y fuerzas políticas con la idea de dar vida a una única estructura lo más unitaria posible, política y económicamente. En 1934 contaba en Asturias con una red bastante amplia de comités, muchos de los cuales, después de la insurrección, quedaron "casi deshechos" ${ }^{33}$. Los comités locales eran nueve y 520 los militantes ${ }^{34}$. En los primeros meses de 1935, con la durísima represión que siguió a la revolución de octubre,la mayor actividad de asistencia correspondió al $\mathrm{SR}^{35}$ y Asturias se

\footnotetext{
30 Ibidem.

${ }^{31}$ Ibidem.

32 "Informe general de la situación de la sección española del Socorro Rojo Internacional después del movimiento insurreccional de octubre", Madrid, 16 de marzo de 1935, firmado por el Comité Ejecutivo Nacional, Secretariado, en AHPCE, film XIV, apartado 171, pág. 4.

33 "Informe general de la situación de la sección española del Socorro Rojo Internacional después del movimiento insurreccional de octubre", Madrid, 16 de marzo de 1935, firmado por el Comité Ejecutivo Nacional, Secretariado, en AHPCE, film XIV, apartado 171.

34 Ibídem; Erice, Francisco (coord), Los comunistas en Asturias 1920-1982, op. cit., pág. 68.

35 "Su labor asistencial contribuyó además a aumentar la popularidad de los comunistas", Ídem.
} 
confirmó como «la región donde más actividad parece haber desarrollado, extendiendo por entonces sus secciones locales» ${ }^{36}$.

Indudablemente, uno de los rasgos novedosos de la política solidaria kominterniana en los primeros meses de 1935 fue el puente tendido hacia distintas tendencias políticas, entre las cuales estaban también las organizaciones republicanas. La campaña de solidaridad organizada por el SR en defensa de los presos, los perseguidos y los condenados a muerte tuvo la ventaja de llegar a "desbordar los márgenes de los partidos obreros" ${ }^{37}$. Fue en el mes de marzo de 1935 cuando la política aliancista dio sus resultados más concretos con la constitución del Comité Nacional de Ayuda a las Víctimas de Octubre ${ }^{38}$. Su creación fue gestionada anteriormente desde París ${ }^{39}$ y luego en una serie de reuniones en el Ateneo de Madrid $^{40}$, gracias al acuerdo entre la IC, el dirigente socialista Álvarez del Vayo y, posiblemente, el miembro del Comité Central del PCE, Vicente Uribe ${ }^{41}$. El SRI celebró su puesta en marcha en la medida en que había sido una iniciativa propia y del $\mathrm{PCE}^{42}$. La función primaria del CNA fue el reparto de las primeras ayudas recibidas de la solidaridad internacional $\left(72.000\right.$ pesetas $\left.^{43}\right)$ y luego las que se fueron recaudando ${ }^{44}$. En la falta de decisión socialista resultó decisiva la escasa disponibilidad financiera de la Federación Internacional de Solidaridad (FIS) que llevó a los socialistas a la inmovilidad de acción en el plano económico y financiero ante los hechos de Asturias ${ }^{45}$.

La organización se apoyó en recursos que se podrían denominar "movilizadores", entre los cuales corresponde un lugar privilegiado a la primera de las Campañas de Navidad e Invierno por el amplio respaldo social que logró desde finales de 1934. Entre los recursos de recaudación que tenían un carácter más visual recordamos la edición de carteles, sellos, postales, viñetas ${ }^{46}$, tarjetas

\footnotetext{
36 También subrayaba entre las organizaciones juveniles a la Federación Cultural Deportiva Obrera (fundada en Asturias en 1933) que desarrollaba una labor de captación y aproximación a los socialistas. Ibídem, pág. 59.

37 Bizcarrondo, Marta, "De las Alianzas Obreras al frente popular", en Estudios de Historia Social, en S. CASTILLO (coord.), Estudios de historia de España. Homenaje a Manuel Tuñón de Lara, Madrid, UIMP, 1981, vol. 2, págs. 95-112, pág. 97.

38 Sobre la fecha de su fundación, véase el «Informe» al que he hecho referencia (AHPCE, film XIV, apartado 171, págs. 3-4), donde se remonta a marzo de 1935, como también lo atestiguan Manuel Tuñón de Lara y Marta Bizcarrondo, mientras que Rafael Cruz, que habla profusamente del CNA, no nos indica la fecha exacta de su fundación, pero aporta otras informaciones relevantes sobre su constitución. Bizcarrondo, Marta, "De las Alianzas Obreras al frente popular", y Tuñón de Lara, Manuel, "El Bloque popular antifascista", en Estudios de historia social, op. cit., pág. 126, y pág. 97; Tuñón de Lara, Manuel, Tres claves de la Segunda República, op. cit., pág. 326; Cruz, Rafael, El Partido Comunista de España en la II República, op. cit., págs. 223-224.

${ }^{39}$ Cruz, Rafael, El Partido Comunista de España en la II República, op. cit., pág. 223, n. 19.

${ }^{40}$ Bizcarrondo, Marta, "Democracia y revolución en la estrategia socialista de la II República", en Estudios de Historia Social, Madrid, Ministerio de Trabajo y Seguridad Social, enero-junio de 1981, núm.16-17, págs. 227- 461, pág. 329.

${ }^{41}$ De la presencia en París de Vicente Uribe para organizar la solidaridad nos informa una vez más Cruz a través de un artículo del entonces dirigente comunista Fernando Claudín sobre el líder vasco. Nuestra Bandera, núm. 31, julioagosto de 1961, págs. 53-56; Cruz, Rafael, El Partido Comunista de España en la II República, op. cit., pág. 223, n. 19.

42 Véase la noticia de la constitución del CNA “a instancias e iniciativas del Socorro Rojo" en VVAA, Historia del Partido Comunista de España, París, Éditions Sociales, 1960, pág. 93; noticia ulteriormente confirmada en SRI, 19231938. XV Años de Solidaridad en España. Labor de Ayuda y Finanzas. Socorro Rojo de España. Conferencia Nacional de Solidaridad 1 y 2 de noviembre de 1938, pág. 1.

43 "Informe general de la situación de la sección española del Socorro Rojo Internacional después del movimiento insurreccional de octubre”, Madrid, 16 de marzo de 1935, Comité Ejecutivo Nacional, Secretariado, pág. 4.

44 “[...] más tarde se empezaron a efectuar suscripciones, alcanzándose grandes sumas, sólo en la parte de Guipúzcoa se recolectaron medio millón de pesetas". SRI, 1923-1938 XV Años de Solidaridad en España. Labor de Ayuda y Finanzas. Socorro Rojo de España. Conferencia Nacional de Solidaridad, 1 y 2 de noviembre de 1938, p. 2.

${ }^{45}$ Bizcarrondo, Marta, "Democracia y revolución en la estrategia socialista de la II República", en Estudios de Historia Social, op. cit., pág. 328.

46 Se crearon una serie de viñetas para la celebración del primer aniversario de octubre de 1934 que animaban nostálgicamente a la conmemoración de la revolución y a la vuelta a la unidad perdida, "Escenas del aniversario de la revolución para conmemorar el primer aniversario de octubre”, en AHPCE, film XIII, apartado 164.
} 
ilustradas y carteles murales ${ }^{47}$ que tuvieron un carácter a menudo muy simbólico y que fueron de amplia aplicación y de gran éxito movilizador.

Una de las prioridades y finalidades del SR fue la ayuda jurídica a los detenidos en 1934. En diciembre de ese año, creó por iniciativa propia la Agrupación de Abogados Defensores de los Encartados por los Sucesos de Octubre (ASO), que constituía una "organización de abogados antifascistas" salvar de la muerte a los encarcelados", además de "pedir para ellos el indulto" jurídicas de la ASO se encargaron de la defensa de los presos, de la asistencia económica, legal y moral a los detenidos políticos en las cárceles y a sus familias fuera de las mismas. En apoyo a la labor judicial patrocinó la realización de varias actividades: recogidas de firmas, como la de agosto de 1935 que alcanzó un éxito enorme $e^{50}$, y grandes campañas nacionales. Las que se emprendieron para salvar de la muerte al dirigente socialista Ramón González Peña, encarcelado el 3 de diciembre de 1934 (indultado junto con otros 19 condenados) ${ }^{51}$, y al comunista Juan José Manso (responsable comunista en el Comité revolucionario de Asturias) tuvieron una resonancia nacional e internacional.

Asimismo fue muy importante la labor de asistencia a la infancia a través de los "Comités ProInfancia Obrera" y a los detenidos con los "Comités Pro-Amnistía". La principal bandera de la política unitaria del ComitéNacional de Ayuda y del SR fue la campañapor la amnistía que tuvo su comienzo oficial en un gran mitin organizado en el cine Europa de Madrid ${ }^{52}$. Para soslayar el problema que suponía la ilegalidad de la organización, se propuso cambiar el nombre de los comités pro amnistía por el de comités contra la pena de muerte o por la liberación de los presos a fin de trabajar dentro de la legalidad ${ }^{53}$.

A esta amplia labor de protesta y movilización siguió una constante actividad de asistencia a los detenidos en las cárceles y se añadió la asistencia a los emigrados políticos que fue encomendada en su totalidad al SR. El SR se cimentó, además, en la importante organización, de manera especial en Asturias, de las redes de fugas, que dieron prueba de gran eficacia mediante el envío de represaliados principalmente a Francia y a la Unión Soviética. Alrededor de dos o tres centenares de trabajadores y dirigentes destacados del movimiento de octubre lograron huir de Asturias. El SRF activó en Francia un vasto dispositivo de ayuda dirigido a los refugiados españoles, cuando el movimiento migratorio desde España se hizo muy intenso, principalmente en el sur de Francia ${ }^{54}$. Entre doscientos y trescientos exiliados cruzaron la frontera francoespañola; mayoritariamente eran asturianos, y les seguían un par de centenares de vascos,

\footnotetext{
47 Se pueden ver ejemplos en. postales editadas por el Secours Rouge Internacional para la liberación de los detenidos políticos: Arrighi, Víctor y Bénard y Clerc, Gaston, AN, série F7, "Secours Rouge”- Bureau Latin - 13154. Secours Rouge International 1921-1929 y Andrè Marty, en AN, série F7, "Secours Rouge" 13515 - Dossier: "SRI 1930 y maifevrier 1931"; sellos para la recaudación del SRF y postal en favor de la liberación de Andrè Marty, en AN, série $\mathrm{F}^{7}$, "Secours Rouge" 13515 - Dossier: "SRI 1930 y mai-fevrier 1931"; o viñeta "Escenas del aniversario de la revolución para conmemorar el primer aniversario de octubre”, en AHPCE, film XIII, apartado 164.

48 SRI, 1923-1938 XV Años de Solidaridad en España. Labor de Ayuda y Finanzas. Socorro Rojo de España. Conferencia Nacional de Solidaridad, 1 y 2 de noviembre de 1938, pág. 1.

49 Boletín Anual de Abogados defensores de los encartados por los sucesos de Octubre, enero de 1936, Madrid, pág. 1.

50 SRI, 1923-1938. XV Años de Solidaridad en España. Labor de Ayuda y Finanzas. Socorro Rojo de España. Conferencia Nacional de Solidaridad, 1 y 2 de noviembre de 1938, pág. 1.

51 "Es una grandiosa victoria contra la reacción”. ¡Combate! Órgano del Socorro Rojo Internacional (SE), abril de 1935, año II, núm. 4 (segunda época), editado por el Socorro Rojo Internacional, p. 8, en AHPCE, film XIV, apartado 171.

52 Socorro Rojo de España (CEN), Memoria sobre las actividades del SRI. Conferencia Nacional de Solidaridad, 1-2 de noviembre de 1938, Madrid, en AHPCE, tesis, manuscritos y memorias, 28.5.

53 Ibidem.

${ }^{54}$ Sobre el significado de la emigración política, sobre todo en relación con Francia, es interesante el capítulo de Groppo, Bruno, "La figura dell' emigrato politico", en Dreyfus, Michel et al., Il secolo dei comunismi, Milano, Net, 2004, págs. 431-446.
} 
madrileños y catalanes, aunque la procedencia geográfica parece ir diversificándose según se fue acercando el año 1935. La primera acción de gran envergadura en el ámbito de la solidaridad hacia los españoles fue la constitución en París del Comité Populaire d'Aide a toutes les Victimes du fascisme en Espagne (CPAV) ${ }^{55}$ el 7 de noviembre de $1934^{56}$.

Así pues, a finales de 1935, parte de los objetivos que se había prefijado la organización kominterniana llegaron a buen fin. El SR logró una mayor pluralidad en la composición de los cuadros y en sus afiliados; asumió, sin lugar a dudas, el papel principal en la defensa de los derechos de los detenidos y luego en la petición de responsabilidades y reparaciones en el ámbito del CNA. Favoreció de paso ${ }^{57}$, o quizá de forma más consciente ${ }^{58}$, o de manera muy modesta según nuestra opinión - la dinámica unitaria que se presentó en 1936 y la definición del Frente Popular ${ }^{59}$.

Es en el mitin del 29 de febrero de 1936 celebrado en Madrid y en el júbilo que se propagaba de Asturias a París, Dieppe, Bruselas y la Unión Soviética tras la aprobación del decreto de amnistía (el 21 de febrero) cuando podríamos ver el acto de clausura de una etapa muy significativa de la solidaridad nacional e internacional. Asimismo, en la salida de las cárceles de los detenidos y en la súbita vuelta de los refugiados políticos se entrevé un logro, además de político, sin duda también solidario. Madrid fue, pues, el lugar de este gran "acto unitario" organizado por el SR, el CNA, el Comité de Amnistía y la Agrupación de Abogados Defensores ${ }^{60}$. Un acto al que siguieron muchos más, como el mitin organizado por el SR y en el que participaron el Partido Socialista, representado por Julio Álvarez del Vayo, Dolores Ibárruri y Juan José Manso, por el PCE, y Esteban Vega, en calidad de secretario del $\mathrm{SR}^{61}$.

\section{El Socorro Rojo, un capítulo del antifascismo femenino: desde Asturias a la guerra civil}

La preponderancia de las actividades político-solidarias llevadas a cabo por las mujeres en el ámbito del Socorro Rojo Internacional fue evidente. El carácter revolucionario y político que adquiere, entre los años veinte y treinta del siglo veinte, la solidaridad, queda reflejado en la movilización de las mujeres. Lejos de la herencia de un concepto de solidaridad de tipo "asistencial", de matriz decimonónica, y alimentado por los valores del siglo XIX, se afirmaba una idea de solidaridad muy concienciada de su nueva fuerza y de su función política. Una solidaridad "combatiente", "activa" y "generosa" como la definía la histórica dirigente del PCE

\footnotetext{
${ }_{55}$ Denominado en la prensa y la documentación españolas más comúnmente como el Comité Popular o el Comité Popular francés Pro-Víctimas de la represión española.

56 Préfecture de Paris, Direction de l'Administration et de la Police Générales. Service des Affaires de Sûreté Générale, les Bureau. Objet "A.S. de l'assistance fournie par les organisations du Secours Rouge Internationale". Firmado por el "Directeur le Commissaire de Police", París, 27 de febrero de 1935, en Archives de la Préfecture de Police (París), ESPAGNE BA (II parte) Ba Comité d'Aide aux réfugiés espagnols (1934-1939), Dossier: "Réfugies politiques espagnols en France 1934 -1935".

57 Erice, Francisco (coord.), Los comunistas en Asturias 1920-1982, op. cit., pág. 71.

${ }^{58}$ El CNA constituyó “el elemento que por nueve meses fue una prefiguración del Frente Popular”, Tuñón de Lara, Manuel, Tres claves de la Segunda República, la cuestión agraria, los aparatos del Estado, Frente Popular, op. cit., pág. 325.

59 Branciforte, Laura "El Socorro Rojo Internacional: agente político de la dinámica unitaria del Frente Popular (1933-1936)", en Congreso Internacional, La España del Frente Popular. Política, sociedad, cultura y conflicto en la España de 1936, Universidad Carlos III de Madrid, 15 y 16 de febrero de 2011, CD ROM.

60 "Las invitaciones se podían recoger en las sedes socialistas y en el local de la Federación Tabaquera, por lo que se puede considerar un acto unitario”. Souto Kustrín, Sandra, “¿Y Madrid?¿Qué hace Madrid?”. Movimiento revolucionario y acción colectiva (1933-1936), Madrid, Siglo XXI, 2004, pág. 394.

${ }^{61}$ Anónimo español (S. XX): Cartel del "Gran mitin de júbilo por la liberación de los presos, organizado por el Socorro Rojo Internacional, Pro-Infancia e Izquierda Republicana Femenina” [Alicante, s.n., 1936], en Jaramillo Guerreira, Miguel Ángel (dir.), Carteles del Archivo General de la Guerra Civil Española, Madrid, Ministerio de Educación, Cultura y Deporte, D. L., 2002, vol. I.
} 
Dolores Ibárruri cuando se refería a la ayuda internacional de las mujeres durante la guerra civil. En especial, es a partir de mediados de los años treinta, cuando la solidaridad se convirtió en sinónimo de acción femenina en el ámbito de la solidaridad antifascista internacional.

El antifascismo favoreció la incorporación de un sector significativo de mujeres en este nuevo ámbito de confrontación política que, a partir de la celebración del Rassemblement mondial des femmes contre la guerre et le fascisme, celebrado en París entre el 4 y el 7 de agosto de $1934^{62}$ tuvo su bautismo en el asociacionismo internacional femenino. Mientras tanto en España se había constituido en 1933 el "Comité Nacional de Mujeres contra la guerra y el fascismo", desde donde se eligió y salió para París un amplio comité de representantes, con Dolores Ibárruri al frente, y que contribuyó a la extensión de redes antifascistas femeninas, como fue, por ejemplo, el caso de la Agrupación de Mujeres Antifascistas (AMA) ${ }^{63}$, que fue la organización en que se convirtió el Comité contra la Guerra y el Fascismo durante la Guerra Civil. Esta asociación fue la demostración práctica de una participación interclasista, además de "interpartidista", y fue, sobre todo, expresión de la puesta en marcha de una participación directa y activa de las mujeres en la política y no sólo en los asuntos del antifascismo nacional e internacional ${ }^{64}$.

Pese a que sea ficticio y algo difícil establecer una línea de demarcación entre las labores de mujeres en la retaguardia y las de hombres en primera línea, es verdad que la labor de "segunda línea" coincidió en las guerras con los roles diariamente desempeñados por el sexo femenino: trabajos de intendencia y tareas definidas como "auxiliares" (abastecimiento, asistencia sanitaria, trabajos "de relevo" de las informaciones), siempre que así se puedan considerar por su fatiga y su peligrosidad y por la amplia y explícita implicación política.

Ahora bien, volviendo al SR, si bien es verdad que las labores asistenciales y el cuidado de los niños han sido preferentemente una prerrogativa femenina, las cifras de la presencia de las mujeres en el Socorro Rojo no parecen responder, hasta mediados de la década de 1930, a esta realidad tan fácil de suponer.

El porcentaje de las mujeres, además, no adquirió en el SR a partir de 1934, y sobre todo desde 1935, un carácter relevante sino más bien lo contrario, mientras que sus acciones sí. Entre 1934 y 1935 la presencia femenina redobló la del año anterior, sin embargo, llegó sólo a un $10 \%{ }^{65}$. Además hay que tener en cuenta que el criterio de afiliación no se correspondió con la presencia real de sus afiliados, dado que se consideraban sólo los cotizantes y hubo una participación y ayuda esporádica y externa difícilmente cuantificable.

Más allá de los datos numéricos, la experiencia de algunas mujeres que sí tuvieron una función destacada en el SR durante estos años ha caído en cierto anonimato y poco o nada se conoce de ellas. Esta situación se debe en buena medida a la escasa capacidad de los partidos para dar relevancia a sus representantes femeninas.

\footnotetext{
${ }^{62}$ Comité contre la guerre et le fascisme, Rassemblement mondial des femmes! Contre la guerre et le fascisme. Compte rendu des travaux du Congrès Paris, les 4, 5, 6, 7 août 1934.

${ }^{63}$ Nash, Mary, Mujer y movimiento obrero en España. 1931-1939, Fontamara, Barcelona, 1984; Yusta Rodrigo, Mercedes, Madres coraje contra Franco. La Unión de Mujeres Españolas en Francia, del antifascismo a la Guerra Fría (1941-1950), Madrid, Cátedra, 2009.

64 A finales de agosto la Agrupación de Mujeres contra la Guerra y el Fascismo, luego AMA, organizó una importante manifestación en Madrid contra el proyecto del gobierno de movilización de reservistas y poco después realizó otra en Zaragoza. Entre el verano y octubre de 1934, el Congreso Nacional realizó una campaña de movilización de las mujeres y se formaron nuevas agrupaciones de la organización. Nash, Mary, Mujer y movimiento obrero en España. 1931-1939, Barcelona, Fontamara, 1984, pág. 243.

65 "Informe general de la situación de la sección española del Socorro Rojo Internacional después del movimiento insurreccional de octubre", Madrid 16 de marzo de 1935, firmado "por el Comité Ejecutivo nacional, el Secretariado", en AHPCE, Film XIV, apartado 171.
} 
Desde 1935, contrariamente a las cifras, empezamos a encontrar los primeros nombres de dirigentes locales del SR en Asturias que coincidían en parte con las encargadas del Partido Comunista Asturiano: Concha González Madera, Oliva López ${ }^{66}$, Rosario Casanueva Vallina, "la Pasionaria de Noreña" ${ }^{97}$, o del Comité Nacional: Encarnación Fuyola y Matilde Landa. La preeminencia de mujeres se detecta también en las acciones más peligrosas, en las noticias de sus decesos (Aída la Fuente ${ }^{68}$ ), de sus condenas, o de las torturas y los sufrimientos padecidos (Maruja de la Fuente) ${ }^{69}$.

El compromiso que el SR y sus mujeres experimentaron con la población española lo volvieron a vivir al cabo de pocos años, durante la guerra civil, cuando la organización acabó constituyéndose en la verdadera base del sistema sanitario militar republicano y cuando, por su relevancia política y su importancia en la ayuda a la población civil, se empezó a conocer más extensamente la acción desarrollada por este organismo y sus protagonistas.

Según iba cobrando importancia el SR empezaron a figurar a su cabeza, por primera vez, los nombres de acreditados representantes del mundo político masculino: Isidoro Acevedo, Luis Zapirain y Esteban Vega en calidad de su presidente y de secretarios del SR. Sin embargo, fueron en realidad dos mujeres -Matilde Landa y Tina Modotti (a) Maria- las verdaderas responsables del SRI y las que ejercieron su dirección en España. Ellas fueron las que, junto a todas aquellas más conocidas, como Encarnación Fuyola, o Elisa Risco, María Luisa Lafíta, Mary Bingham Urquidi, Flor Cernuda,y las menos conocidas, como Amelia Álvarez Díaz o Elvira FernándezAlmoguera ${ }^{70}$, entre muchas, trabajaron además de en las tareas de organización, en las arduas y urgentes tareas de ayuda y de socorro que la guerra les iba presentando ${ }^{71}$.

La maquinaria del SR se puso en marcha al día siguiente de que estallase la sublevación militar con la constitución de una Comisión de Solidaridad ${ }^{72}$, dirigiéndose de inmediato a sus principales protagonistas, las mujeres, con un primer llamamiento realizado por radio para buscar enfermeras -tituladas o no- y donantes de sangre ${ }^{73}$. La respuesta fue inmediata y masiva: cinco mil mujeres ofrecieron su concurso ${ }^{74}$.

En la guerra civil, la denominada "Cruz Roja del antifascismo del Frente Popular" "75 se encargó de hacer frente a las imperantes necesidades de los milicianos y de la población civil y fue entonces cuando la historia del Socorro Rojo se convirtió ya en una historia preponderantemente de

${ }^{66}$ La presidencia del SR en Gijón fue encargada a una mujer, Oliva López, Secretaría del Comité Local del PCE., Fernández Riera, Macrino, Mujeres de Gijón (1898- 1941), ed. KRK, Gijón, 2006, pág. 348.

${ }^{67}$ Cabrero Blanco, Claudia, Mujeres contra el franquismo. (Asturias 1937-1952), KRK ed., Oviedo, 2006, pág. 213.

${ }^{68}$ Murió "asesinada de forma criminal" cuando actuaba como enlace entre el Comité Revolucionario de Oviedo y los grupos en el Oeste de la ciudad el día 14 de octubre de 1934 a la edad de 16 años, "cubriendo la retirada del ejército rojo de Asturias". "Informe sobre la Revolución en Asturias de 1934 y comités prerrevolucionarios provinciales" Capítulo XXXII: "Nuestro servicio de espionaje", págs. 113-120 en AHPCE, Nacionalidades y Regiones (A), caja 79, carpeta 3.3.; "Una heroína española", ;Ayuda! Portavoz de la solidaridad, op. cit., pág. 7.

${ }^{69}$ La hermana de Aída, Maruja, "fue detenida por los soldados, le arrancaron todas las ropas del cuerpo, le ataron los brazos a la espalda, y así, completamente desnuda, la condujeron hasta frente a un muro, donde había de ser fusilada...". "Una heroína española" iAyuda! Portavoz de la solidaridad, Año I- Núm.4, Madrid, 15 de marzo de 1936, pág. 7.

${ }^{70}$ Presidenta del Socorro Rojo Internacional de Albacete

${ }^{71}$ Branciforte, Laura, El Socorro Rojo Internacional en España (1923-1939). Relatos de la solidaridad antifascista, Madrid, Biblioteca Nueva, 2011, págs. 18-21.

${ }^{72}$ De ella formaban parte las organizaciones antifascistas nacionales. Esta comisión mantuvo las relaciones con los organismos internacionales de ayuda a España hasta que el abril de 1938 el gobierno creó el Comité de Ayuda a la España Republicana, presidido por Martínez Barrio. Ibídem, pág. 13.

${ }^{73} \mathrm{El} \mathrm{CE}$ del SR tuvo en principio una emisora radiofónica propia la E.A.J.4, "la emisora de la solidaridad", hasta que las medidas del gobierno lo impidieron y empezó a usar emisiones regulares. Ibídem, pág. 18.

74 “[...] solo el primer día acudieron 3000 mujeres". Ibídem, pág. 5.

75 "Resolución del Comité Ejecutivo Mundial del SRI sobre el Socorro Rojo de España" firmado por el Comité Ejecutivo Mundial del S.R.I. Servicio Histórico Militar (SHM), Rollo 275, Legajo 77 bis, carpeta 7, doc. 2 pág. 1. 
mujeres. Fueron ellas las que se encargan de la acción solidaria y política en los campos de batalla y en las retaguardias, de la recogida de los heridos, del abastecimiento de víveres y ropas para los combatientes, de la organización de provisión de ambulancias, de las camillas o de la organización de hospitales de sangre ${ }^{76}$. Fueron ellas las enfermeras, a las que se les encomendó las evacuaciones de las ciudades, así como las que cubrieron la arriesgada función de "enlace" para hacer llegar informaciones.Y fueron ellas también las que asistieron a los milicianos heridos y a sus familias, y las que atendieron la seguridad de los hospitales frente a posibles infiltraciones de quintacolumnistas.

No hay que olvidar que era un momento en el que la organización sanitaria militar era, sin duda, muy compleja y vivía momentos caóticos de reorganización y sustitución de un aparato hasta entonces prerrogativa del Estado. Después del golpe militar y el sucesivo inicio de la guerra civil, los aparatos del Estado republicano se vieron desamparados y resquebrajados. Como denunciaba un informe del SR: "la mayoría de los componentes de la Sanidad y de la Intendencia militar eran monárquicos y fascistas" "77. El SR, a través de su Comisión Nacional de Sanidad, dio vida a un nuevo sistema sanitario militar, bien estructurado, y logró construir 275 "hospitales de sangre", instaló fábricas de camillas y artolas, proveyó la Sanidad militar de ambulancias y rayos $\mathrm{X}^{78}$, y colaboró con la sanidad civil, haciendo frente a la urgencia bélica hasta la sucesiva y gradual centralización de la gestión del sistema sanitario a partir de 1937.

La gran utilidad de esta labor desarrollada por el SR fue inmediata y oficialmente reconocida por la Jefatura de Sanidad del Ejército republicano ${ }^{79}$.Mientras tanto, el avance de la guerra imprimía nuevas prioridades al SR, como la de acoger a los refugiados que procedían de los territorios ocupados de las provincias de Toledo ${ }^{80}$, Cáceres y Ávila. A tal propósito se creó una Comisión Nacional de Abastos del SRI en Madrid ${ }^{81}$ con las tareas de coordinar y redistribuir la llegada de víveres y ropa que, en su mayoría, procedían de Levante y Cataluña ${ }^{82}$. El SR empezó a organizar centros de avituallamiento y refugios para los evacuados en Madrid: "antes se utilizaron las casas deshabitadas, luego se crearon centros de acogida". El más grande fue el ubicado en los "pabellones de la Ciudad Universitaria" 83 .

En diciembre de 1936 las oficinas del CEN del SRI fueron trasladadas a Valencia, donde ya había llegado su presidente, Isidoro Acevedo, en el mes de octubre ${ }^{84}$. La nueva sede se encontraba en la

\footnotetext{
76 "Socorro Rojo Internacional". Labor de Ayuda y finanzas: 1923-1938, Madrid, Comité Ejecutivo Nacional (1938), págs. 2-3

77 Socorro Rojo Internacional, Sección Española, Seis meses de solidaridad Antifascista. Resumen del Pleno nacional celebrado en Valencia los días 7 y 8 de febrero de 1937, Ediciones SRI, 1937, pág. 7. El SRI “organizó la Sanidad militar, abandonada y desorganizada por los facciosos, ayudó enormemente a la creación de una intendencia militar sólida". Contreras, Carlos "Las tareas fundamentales de Socorro Rojo Español", en Ayuda. Semanario de la solidaridad, año II, núm. 55, Madrid, 16 de mayo de 1937.

78 Socorro Rojo Internacional, Sección Española, Seis meses de solidaridad Antifascista, op. cit., pág. 7.

79 "Documento de la Jefatura de Sanidad del Ejército republicano", Valencia, 11 de marzo de 1937, firmado por el Jefe de Sanidad Militar republicana. Biblioteca Nacional de España, Madrid.

${ }^{80}$ Destacó la labor del SR en la evacuación de los heridos desde Toledo para la cual realizó la instalación de 500 camas en un plazo de horas tras la petición de la Jefatura de Sanidad. "Documento de la Jefatura de Sanidad del Ejército republicano", Valencia, 11 de marzo de 1937, firmado por el Jefe de Sanidad Militar republicana. En Biblioteca Nacional (BNE), Madrid.

${ }^{81}$ En esta comisión, la participación del SR estuvo dirigida por la viuda de Luis de Sirval, María Rubio. "El Socorro Rojo al lado de los combatientes populares", La Vanguardia, sábado 20 de febrero de 1937.

82 Ibídem.

83 Socorro Rojo de España. CEN, Memoria sobre las actividades del SRI y ;Ayuda! Semanario de solidaridad, año I, núm. 24 , Madrid, 31 de octubre de 1936.

84 "El día 9 de Octubre de 1936 el Partido me ordenó que saliese inmediatamente de Madrid para Valencia, donde proseguí mi labor en el Socorro Rojo”. “Autobiografía de Isidoro Acevedo”, en AHPCE, dirigentes, sig. 1 / 2 .1.1.
} 
calle Márquez de Montornés, como relataba Fanny Edelman ${ }^{85}$. Tina Modotti y Matilde Landa, verdaderas protagonistas de la dura labor del Socorro Rojo en España,se marcharon también a Valencia, nueva sede del gobierno y de los ministerios, y ciudad relativamente más segura por el momento. Antes de dejar Madrid, a Modotti y Landa les tocó presenciar la evacuación del Hospital de Maudes, desde el cual los heridos fueron trasladados a Valencia y Albacete. Al mes, Modotti participó en la organización del Pleno Nacional del SR en Valencia, celebrado los días 7 y 8 de febrero con la asistencia de 44 delegados de 19 provincias y el SR de Cataluña. En este pleno, que se realizó justo después de la toma de Málaga, se acordó entregar al gobierno de Francisco Largo Caballero todo lo que hasta entonces se había recabado en el ámbito de la sanidad e intendencia ${ }^{86}$ y se decidió dirigir la atención y los esfuerzos mayoritariamente al socorro de los evacuados ${ }^{87}$. La aterradora y urgente realidad de la evacuación de Málaga imponía nuevas prioridades a la organización.

Como comentaba Tina Modotti, la peor experiencia de su vida, "aún más terrible que la de Madrid", fue la que vivió entre Málaga, Motril y Almería junto a Matilde Landa y el célebre doctor Norman Bethune, con el que ya habían trabajado en Madrid con su servicio canadiense de transfusiones de sangre ${ }^{88}$. Con motivo de la estratégica toma de Málaga y de su puerto el 6 de febrero de 1937, los esfuerzos del SR junto con las instituciones estatales, "el Consejo de Sanidad, primero, y el Consejo provincial, más tarde, se volcaron a estructurar la nueva red asistencial", a la evacuación por la carretera Málaga-Almería y a la asistencia a la población. El ataque a la capital almeriense el 12 de febrero obligó a trasladar a la "casa-refugio" del pueblo de Vera al personal médico y a los heridos ${ }^{89}$, y a evacuar a la población civil desde Almería y alrededores hacia el arco levantino republicano y Cataluña. Pese a que, por supuesto, el SR no fue el único agente de la acción médica y solidaria, seguramente fue uno de los momentos en los que su intervención destacó ante la urgencia de los evacuados. De esta realidad se ocupó de forma conjunta con el Ministerio de la Presidencia y, a partir de enero de 1937, con el Ministerio de Sanidad y Asistencia Social bajo la dirección, hasta el nuevo gobierno presidido por Juan Negrín en mayo de 1937, de Federica Montseny ${ }^{90}$.

Desde que la guerra se había extendido por todo el territorio nacional aumentaron las demandas y las respuestas de la solidaridad internacional. El devenir de la guerra obligó a la celebración

${ }^{85}$ Fanny Edelamnn decía: "Con ella me unió una fuerte amistad, inolvidables charlas. Salíamos juntas de nuestro alojamiento en la Calle Conde de Carlés, hacia la Sede del Socorro Rojo, en la calle Márquez de Montornéz. [sic] y regresábamos, un poco amparándonos una a otra, tomadas del brazo y yo enmudecía ante la lucidez de aquella mujer con tanta valentía y sensibilidad", en Calloni, S., "Fanny Edelman, 70 años de militancia", La Jornada, México, 27 y 28 de septiembre de 2004.

86 "Cuando la Sanidad creyó oportuno centralizar toda la Sanidad de Guerra en el Ejercito, el SR pudo ofrecerle: 275 hospitales, equipo de trasfusiones de sangre, escuela de enfermeras y camilleros en Madrid, fábrica de ambulancias en Madrid, fábrica de camillas y artolas en Valencia, almacén de instrumental y material sanitario y un gran plantel de médicos practicantes y enfermeras experimentados". En CEN, Memoria sobre las actividades del SRI, op. cit., pág. 10.

87 "El Pleno pone en lugar destacado de las actividades del SR las actividades [...] en la retaguardia, con el fin de acoger a los millares de evacuados, especialmente mujeres y niños". Resoluciones aprobadas por el Pleno Nacional del Socorro Rojo de España, Valencia, 7 y 8 de febrero de 1937, SHM, legajo 77 bis, capeta B, doc.2.

88 Gracias a él las transfusiones de sangre en el frente pasaron a ser un punto de referencia en la historia médicomilitar de la guerra civil; Bethune volvió a Canadá, donde siguió recaudando fondos para España. En 1938 se fue a China, donde al año siguiente murió. Véase Norman Bethune. El crimen de la carretera Málaga-Almería (febrero de 1937), Benalmádena (Málaga), 2004; Allan, Ted; Gordon, Sydney, The Scalpel, The Sword. The Story of Dr. Nornman Bethune, Toronto, Mc-Clelland and Stewart, 1952. Sobre la introducción y organización en España del sistema de las unidades móviles de transfusiones, véase también el documental Hearth of Spain, producido por The Canadian Committee to Aid Spain y The American Medical Bureau to Aid Spanish Democracy, conservado en la Filmoteca Nacional Española, Madrid.

$89 \mathrm{Al}$ poco tiempo el SR se enfrentó a un ulterior traslado del personal y heridos del Hospital de Sangre de Vera a Cuevas de Almanzora al Hospital Militar de Fortuna. Sofía Rodríguez, Mujeres en guerra. Almería 1936-1939, op. cit., pág. 362.

90 Tavera, Susanna, Federica Montseny. La indomable (1905-1994), Barcelona, Temas de Hoy, 2005, pág. 216. 
inmediata de congresos de carácter nacional e internacional para afrontar la situación de emergencia sanitaria y bélica de los frentes republicanos. La creación de la Central Sanitaria Internacional(CSI) ${ }^{91}$ los días 3 y 4 de julio durante la Conferencia Internacional Sanitaria de Ayuda al Pueblo Español, que estableció una delegación permanente en la Jefatura de Sanidad en Valencia, fue una respuesta a la difícil situación padecida por la población ${ }^{92}$.

Mientras tanto, en el mes de julio se iniciaba la organización del conocido II Congreso Internacional de Intelectuales Antifascistas ${ }^{93}$, que tuvo un gran eco internacional. Fue inaugurado el 4 julio en la sala de sesiones del ayuntamiento por el presidente del gobierno republicano, el doctor Juan Negrín, y se desarrolló en el mes de julio entre Madrid (del 5 al 8) ${ }^{94}$, Barcelona (el 11) y París (del 16 y 17) ${ }^{95}$. A pesar de las difíciles condiciones en las que se llevó a cabo, contó con una amplísima presencia del mundo de la cultura y entre los participantes hubo escritores soviéticos, franceses, portugueses, italianos, americanos, españoles: "todo el mundo de la inteligencia que están con los soldados de la República y con toda la España leal [...] André Malraux, Anna Seghers, Hemingway, Aleksei Tolstoi, Octavio Paz y su mujer, Robert Capa y Gerda Taro"96 fueron solo algunos de ellos.

A partir del 7 de noviembre de 1937, adelantando en una semana su tradicional inicio, se puso en marcha, en la fecha del aniversario de la defensa de Madrid, la Campaña de Invierno, en coordinación con el Ministerio de Defensa Nacional, "por conducto y depósito de Intendencia"97. Era uno de los instrumentos más eficaces para la recaudación, y se recolectaron "una serie de prendas accesorias de invierno [...] bufandas, pasamontañas, guantes, calcetines, etc., y mantas" para ayudar a la población de la capital ${ }^{98}$, mientras que la Intendencia de Guerra se ocupó de proporcionar "los uniformes reglamentarios a todo el Ejército" . Esta campaña se distinguió como uno de los episodios más unitarios y de más amplia participación de la solidaridad ${ }^{100}$ : se creó una Comisión Nacional Pro-Campaña de Invierno "cuya presidencia se

\footnotetext{
91 "Se ha conseguido formar, el 4 de julio último, la Central Sanitaria Internacional, que con residencia en París, acumula y centraliza los esfuerzos de todas las Centrales". "Informe sobre la Central Sanitaria Internacional", en SHM, rollo 501, legajo 550, carpeta 4, doc. 9.

${ }^{2}$ La Central Sanitaria Internacional tenía su sede en Rue de Lafayette, 38 bis, mientras que la delegación permanente del CSI en Valencia "designada por la Secretaría del CSI acerca del Gobierno de la República española” estaba ubicada en la Avda. Nicolás Salmerón 4, y el doctor René Michel fue nombrado delegado principal. Ibídem. A la Conferencia había asistido Matilde Landa. "Conferencia Internacional Sanitaria de ayuda al pueblo español", en ¡Ayuda! Semanario de la solidaridad, año II, núm. 64, 18 de julio de 1937.

93 En la sesión inaugural hubo un "Saludo del SRI al Congreso Internacional de intelectuales antifascistas", en ¡Ayuda!, Semanario de la solidaridad, Madrid, 27 de julio 1937, núm. 65.

94 "Los intelectuales del mundo con Madrid", ;Ayuda! Semanario de la solidaridad, año II, núm.65, Madrid, 25 de julio de 1937, pág. 7.

95 Aznar Soler, Manuel y Schneider, Luis Mario, II Congreso Internacional de escritores antifascistas (1937), Barcelona, Laia, 1978.

96 "Los intelectuales del mundo con Madrid", ;Ayuda! Semanario de la solidaridad, año II, núm.65, Madrid, 25 de julio de 1937, pág. 7.

97 "Las entregas se harán en los Parques y Depósitos de Intendencia [...] el plazo máximo de entrega será de diez días a partir de la publicación de esta circular". Circular del Ministerio de Defensa, Valencia, 23 de septiembre de 1937 en AHPCE, carpeta 28.4.

98 Se incluían tres categorías de destinatarios: “a los niños y refugiados, a los combatientes e inválidos de guerra, a la población civil más necesitada". Comité Provincial Pro-ayuda del Socorro Rojo de España, "Campaña pro-ayuda", en AHPCE, carpeta 28.4.

${ }^{99}$ Ibídem.

100 El SRI recaudó para su envío a Madrid 1.500 toneladas de víveres, la mitad del total de las ayudas -entre 6 y 7 millones de pesetas (según las fuentes) - recaudadas en esta ocasión. "1923-1939- A través del Socorro Rojo de España. XV años de Solidaridad”, op. cit., pág. 8. Socorro Rojo de España. CEN. Memoria sobre las actividades del SRI, op. cit., pág. 9.
} 
ofreció al propio Manuel Azaña"101, se contó con el apoyo del poeta Antonio Machado y también -aunque es un hecho aislado- con la colaboración de la Solidaridad Internacional Antifascista $(\mathrm{SIA})^{102}$, que logró ingentes cantidades de víveres, combustible y dinero.

En los primeros meses de 1937 comenzó una vasta acción parael socorro y la adopción desde el extranjero de niños españoles que se habían quedado huérfanos. El 21 de marzo zarpó desde Valencia hacia la Unión Soviética el barco Cabo de Palos con 70 niños españoles a bordo, que llegaron el 30 del mismo mes al puerto de Yalta. En julio, 500 niños salieron de España hacia Ciudad de México: la tarea había sido asumida directamente por Tina Modotti que, en septiembre, pocos meses después de la salida de los pequeños desde España, escribió una conmovedora carta al Comité de Ayuda a los Niños del Pueblo Español consede en la Ciudad de México, en la cual, tras agradecer al pueblo mexicano y a Lázaro Cárdenas su recepción, pedía que se enviasen fotografías de la llegada "de los niños" a México ${ }^{103}$.

A la altura de 1938 la línea de resistencia de la solidaridad kominterniana coincidía perfectamente con la política del Partido Comunista que constituía ya la fuerza más poderosa de la España republicana. La propuesta aprobada por el Comité de No-Intervención para la retirada forzosa y parcial de las tropas voluntarias extranjeras de España, la salida de España de las Brigadas Internacionales a finales de octubre de 1938 y el inicio de la ofensiva contra Cataluña el 23 de diciembre marcaban definitivamente el término de la guerra y el inicio de una sangrienta y larga derrota.

El 26 de enero, día en el que cayó la ciudad de Barcelona, la labor del SR se dirigió a la asistencia a la ola de refugiados que cruzaban los Pirineos hacia Francia, pasando por la dura experiencia del éxodo caótico e infernal junto con decenas de miles de soldados y civiles. María había sido encargada de llevar a París al anciano fundador del Partido Comunista, Isidoro Acevedo, y al comunista italiano Melchiorre Vanni, (a) Bonet, que había dirigido en París el Comité Internacional de Coordinación de la Ayuda para España y que moriría a los pocos días de cruzar los Pirineos. Ella misma logró pasar la frontera después de la caída de Gerona, el 4 de febrero de 1939.

\section{A modo de conclusión}

Podemos decir que a la luz de la acción desarrollada hasta los últimos momentos de la guerra civil por el SR, el estallido de ésta contribuyó tristemente a definir las funciones de la organización, fijándolas, principalmente, en las de asistencia sanitaria y labores de la retaguardia. "Si con alguien trabajaba el SR fue con el gobierno, con el Ministerio de Sanidad durante los años de la Guerra

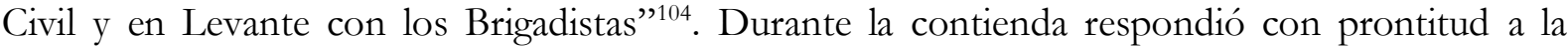
urgencia de la situación. La afluencia en sus filas de nuevos afiliados, y sobre todo nuevas afiliadas y colaboradoras, convirtió al SR en una organización "de masas", como siempre había anhelado ser. Este organismo se transformó, a la vez que el PCE, en una realidad cuantitativa y cualitativamente mucho más presente en el territorio y en la política española y su labor de solidaridad con la República fue inestimable. Fue efectivamente un instrumento de la política de

\footnotetext{
${ }^{101}$ Carta de Luis Zapirain a Isidoro Acevedo, 2 de diciembre de 1937, Socorro Rojo Internacional, Comité Ejecutivo Nacional (SE), Valencia, en AHPCE, Dirigentes, 1/2.2.

102 En abril de 1937 la CNT - a pesar de la oposición de la AIT - redactó los estatutos para la creación de una nueva organización paralela al SR, la sección española de la Solidaridad Internacional Antifascista (SIA). Socorro Rojo, “1923-1939- A través del Socorro Rojo de España. XV años de Solidaridad”, Madrid, 1938, en AHPCE, carpeta, 28.5, pág. 7

103 "Nuestra gran preocupación: los niños", ;Ayuda! Semanario de la solidaridad, año II, núm. 62, Valencia, 4 de julio de 1937. Autoría incierta. ;Ayuda! Boletín del Comité de Ayuda a los niños del pueblo español. Redactado en México D.F.; Carta de María al Comité de Ayuda a los Niños del Pueblo Español de parte del Comité ejecutivo nacional del SRI. Amable donación de Manuel García.

104 SRI, Normas para el funcionamiento del Socorro Rojo de España, op. cit., págs. 6-7.
} 
captación comunista, pero al mismo tiempo fue también un sincero instrumento de solidaridad y, en especial, para las mujeres que, a partir del otoño de 1936, por el decreto de Largo Caballero de reorganización de las milicias populares tuvieron que adecuarse a la consigna «Hombres al frente, mujeres al trabajo» y volvieron a la retaguardia. A partir de ese momento, el gobierno republicano obligaría a la retirada de las milicianas a la retaguardia, apelando a supuestas razones de eficacia militar y de "salud" en el frente, y recurriendo a argumentos sexistas de desprestigio, descalificación y condena moral de las milicianas ${ }^{105}$.

Las mujeres fueron, en todo caso, las protagonistas y portadoras principales de los valores y luchas político-solidarias durante y después de la guerra civil en la que poco afortunadamente se define como la retaguardia, en la resistencia y en el asociacionismo antifascista fuera de España.

105 Tras el decreto de Largo Caballero, en el periódico «Mundo Obrero» de noviembre de 1936 se publicaba un mensaje, bajo la consigna "Mujeres a la retaguardia". "Las mujeres han cumplido su deber [...] Pero ahora el deber principal es reintegrarse a la retaguardia. La marcha de la nación no debe ser interrumpida por la falta de brazos. Esos brazos deben ser suplidos por la mujer", Alcalde, Carmen, La mujer en la guerra civil española, Madrid, Cambio 16, 1976, pág. 125; Sobre este decreto véase, entre otras referencias, Nash, Mary, La Miliciana: otra opción de combatividad femenina antifascista, en Las Mujeres y la guerra civil española, Madrid, Ministerio de Cultura, 1991, págs. 97-108; Aguado, Ana, "Ciudadanas y heroínas antifranquistas: morir por la República española", en Storia delle donne, 4 (2008), págs. 133-151. 